\title{
Three Solutions Theorem for $p$-Laplacian Problems with a Singular Weight and Its Application
}

\author{
Yong-Hoon Lee, ${ }^{1}$ Seong-Uk Kim, ${ }^{2}$ and Eun Kyoung Lee ${ }^{3}$ \\ ${ }^{1}$ Department of Mathematics, Pusan National University, Busan 609-735, Republic of Korea \\ ${ }^{2}$ Department of Mathematics, University of Alabama at Birmingham, Birmingham, AL 35294-1170, USA \\ ${ }^{3}$ Department of Mathematics Education, Pusan National University, Busan 609-735, Republic of Korea
}

Correspondence should be addressed to Eun Kyoung Lee; eunkyoung165@gmail.com

Received 3 September 2013; Revised 18 December 2013; Accepted 1 January 2014; Published 18 February 2014

Academic Editor: Adem Kılıçman

Copyright (C) 2014 Yong-Hoon Lee et al. This is an open access article distributed under the Creative Commons Attribution License, which permits unrestricted use, distribution, and reproduction in any medium, provided the original work is properly cited.

We prove Amann type three solutions theorem for one dimensional $p$-Laplacian problems with a singular weight function. To prove this theorem, we define a strong upper and lower solutions and compute the Leray-Schauder degree on a newly established weighted solution space. As an application, we consider the combustion model and show the existence of three positive radial solutions on an exterior domain.

\section{Introduction}

Let us consider the following $p$-Laplacian problem with a sign-changing singular weight:

$$
\begin{gathered}
\varphi_{p}\left(u^{\prime}(t)\right)^{\prime}+h(t) f(u(t))=0, \quad t \in(0,1), \\
u(0)=0, \quad u(1)=0,
\end{gathered}
$$

where $\varphi_{p}(s)=|s|^{p-2} s, p>1, f \in C(\mathbb{R}, \mathbb{R})$, and $h \in L_{\text {loc }}^{1}((0$, $1), \mathbb{R})$ may change sign. Moreover, $h \in \mathscr{H}$ and satisfies $|h(t)|>$ 0 , for $t \in(0, \delta) \cup(1-\delta, 1)$ for some $\delta>0$, where a class $\mathscr{H}$ of weight functions is given as

$$
\begin{gathered}
\mathscr{H}=\left\{h \in L_{\mathrm{loc}}^{1}(0,1) \mid \int_{0}^{1 / 2} \varphi_{p}^{-1}\left(\int_{s}^{1 / 2}|h(\tau)| d \tau\right) d s\right. \\
\left.+\int_{1 / 2}^{1} \varphi_{p}^{-1}\left(\int_{1 / 2}^{s}|h(\tau)| d \tau\right) d s<\infty\right\} .
\end{gathered}
$$

It is well known that $L^{1}(0,1) \varsubsetneqq \mathscr{H}$.

If $h \in L^{1}(0,1)$, then all solutions of $(P)$ are in $C_{0}^{1}[0,1]$ and based on the Leray-Schauder degree argument on $C^{1}$ space: Ben-Naoum and de Coster [1] proved three solutions theorem for $(P)$. On the other hand, if $h \notin L^{1}(0,1)$, then solutions of $(P)$ may not be in $C_{0}^{1}[0,1]$; for example, take $h(t)=(p-1) t^{-1}|1+\ln t|^{p-2}, p>2$, and $f \equiv 1$, then $h \in \mathscr{H} \backslash L^{1}(0,1)$ and the solution $u$ for corresponding problem of $(P)$ is given by $u(t)=-t \ln t$ which is not in $C^{1}[0,1]$. Thus if $h \in \mathscr{H} \backslash L^{1}(0,1)$, the three solutions theorem in [1] can not be applied. Our main interest in this paper is to establish three solutions theorem for problem $(P)$ for those weights $h$ satisfying $h \in \mathscr{H} \backslash L^{1}(0,1)$.

Main step for the proof of three solutions theorem, in general, is to compute the Leray-Schauder degree on a sector in solution space made from strong sense of upper and lower solutions. Since the sector needs to be open in the space, strong sense of ordering and the sector made from the ordering are closely related to the topology of solution space. A typical situation in application usually happens as follows.

It is comparatively easy to find a lower solution $\alpha$ and an upper solution $\beta$ of $(P)$ satisfying $\alpha(t)<\beta(t)$, for all $t \in(0,1)$ and $\alpha(0)=\alpha(1)=\beta(0)=\beta(1)=0$. Denote $\Omega=\{u \in$ $X \mid \alpha(t)<u(t)<\beta(t)$, for all $t \in(0,1)\}$. In $C^{1}$-analysis, that is, $X=C_{0}^{1}[0,1]$, we see that $\Omega$ is nonempty and open in $X$ by providing additional conditions like $\alpha^{\prime}(0)<\beta^{\prime}(0)$ and $\alpha^{\prime}(1)>\beta^{\prime}(1)$ which implies a strong sense of ordering. On the other hand, in $C$-analysis, that is, $X=C_{0}[0,1]$, we see int $\Omega=\emptyset$ so that the Leray-Schauder degree on $\Omega$ is not 
even defined. Three solutions theorem with no use of such $\Omega$ is very restrictive in application. To overcome this difficulty in our problem, we introduce a weighted solution space, say $C_{w}[0,1]$, which is finer than $C[0,1]$ and also introduce a strong sense of ordering suitable to $C_{w}$-space which makes the degree computation effective (see Section 2 in detail).

As to a question of triple multiplicity of solutions, besides the Amann type three solutions theorems, many works are done by using the variational method (see [2-5] and the references therein) and by using several extensions of the Liggett-Williams fixed point theorem and Guo-Krasnoselskii fixed point theorem, especially for positive solutions (see [6-8] and the references therein). For the problem we are concerned with in this paper, the variational setup is not possible due to lack of regularity of solutions. Three solutions theorem proved in this paper is for the case that $h$ is not only $h \notin L^{1}(0,1)$ but also possibly sign-changing. By this aspect, it is new as far as the authors know. For the case $h \geq 0$, one may refer to [9] for a partial result about the theorem.

As an application, we study the existence of triple positive solutions for certain nonlinear $p$-Laplacian problems with positive singular coefficient function and give an example of a combustion model defined on an exterior domain. Applying this three solutions theorem to the case having sign-changing coefficient function could be an interesting and challenging problem.

We organize this paper as follows. In Section 1, we introduce a weighted solution space $C_{w}[0,1]$, the strong upper and lower solutions of $(P)$, and prove three solutions theorem for problem $(P)$. In Section 3, we prove a multiplicity result for certain nonlinear $p$-Laplacian problems by using three solutions theorem introduced in Section 2. In Section 4, we apply the result in Section 3 to a combustion model to show the existence of three positive radial solutions on exterior domain.

\section{Preliminaries}

In this section, we introduce a weighted solution space $C_{w}[0,1]$ and prove three solutions theorem for $(P)$ on $C_{w}[0,1]$. Let

$$
\begin{aligned}
& C_{w}[0,1] \\
& =\left\{u \in C[0,1] \cap C^{1}(0,1) \mid-\infty<\lim _{t \rightarrow 0^{+}} w(t) u^{\prime}(t)<\infty,\right. \\
& \left.\quad-\infty<\lim _{t \rightarrow 1^{-}} w(t) u^{\prime}(t)<\infty\right\},
\end{aligned}
$$

where

$$
w(t)=\min \left\{w_{h}(t), 1\right\},
$$

with

$$
w_{h}(t)= \begin{cases}\left(\varphi_{p}^{-1}\left(\int_{t}^{1 / 2}|h(s)| d s\right)\right)^{-1}, & 0<t \leq \frac{1}{2}, \\ \left(\varphi_{p}^{-1}\left(\int_{1 / 2}^{t}|h(s)| d s\right)\right)^{-1}, & \frac{1}{2} \leq t<1 .\end{cases}
$$

If $h \in \mathscr{H} \backslash L^{1}(0,1)$, then $0<w(t) \leq 1$, for $t \in(0,1)$,

$$
\lim _{t \rightarrow 0^{+}} w(t)=0 \quad \text { or } \quad \lim _{t \rightarrow 1^{-}} w(t)=0,
$$

and $w^{-1}$ is integrable on $(0,1)$. More precisely, if $h \notin L^{1}(0$, $1 / 2]$, then $\lim _{t \rightarrow 0^{+}} w(t)=0$ and if $h \notin L^{1}[1 / 2,1)$, then $\lim _{t \rightarrow 1^{-}} w(t)=0$.

For $u \in C_{w}[0,1]$, define $w u^{\prime} \in C[0,1]$ by

$$
w u^{\prime}(t)= \begin{cases}\lim _{t \rightarrow 0^{+}} w(t) u^{\prime}(t), & t=0, \\ w(t) u^{\prime}(t), & t \in(0,1), \\ \lim _{t \rightarrow 1^{-}} w(t) u^{\prime}(t), & t=1 .\end{cases}
$$

And also define $\|u\|_{w}=\|u\|_{\infty}+\left\|w u^{\prime}\right\|_{\infty}$; then $\left(C_{w}[0,1],\|\cdot\|_{w}\right)$ is a Banach space. We give a proof for reader's convenience.

Lemma 1. Let $h \in \mathscr{H} \backslash L^{1}(0,1)$; then $C_{w}[0,1]$ is a Banach space with a norm $\|u\|_{w}=\|u\|_{\infty}+\left\|w u^{\prime}\right\|_{\infty}$.

Proof. Let $\left\{u_{n}\right\}$ be a Cauchy sequence in $\left(C_{w}[0,1],\|\cdot\|_{w}\right)$. Then $\left\{u_{n}\right\}$ and $\left\{w u_{n}^{\prime}\right\}$ are Cauchy sequences in $C[0,1]$ so that there exist $u, v \in C[0,1]$ such that $u_{n} \rightarrow u$ and $w u_{n}^{\prime} \rightarrow v$ in $C[0,1]$. It is sufficient to show that $w u^{\prime} \equiv v$ on $[0,1]$. Since $w(t)>0$ for $t \in(0,1)$, there exists $z \in C(0,1)$ such that $z(t)=v(t) / w(t)$ for all $t \in(0,1)$. For $\delta>0$, we know $u_{n}^{\prime} \rightarrow z$ in $C[\delta, 1-\delta]$. This implies $z \equiv u^{\prime}$ in $[\delta, 1-\delta]$. Since $\delta>0$ is arbitrary, $w u_{n}^{\prime} \rightarrow w u^{\prime}$ pointwise in $t \in(0,1)$. Therefore, $w u^{\prime} \equiv v$ on $(0,1)$. Since $w u_{n}^{\prime}$ converges uniformly to $v$ on $[0,1]$, we have

$$
\begin{aligned}
v(0) & =\lim _{n \rightarrow \infty} w u_{n}^{\prime}(0)=\lim _{n \rightarrow \infty} \lim _{t \rightarrow 0^{+}} w(t) u_{n}^{\prime}(t) \\
& =\lim _{t \rightarrow 0^{+}} \lim _{n \rightarrow \infty} w(t) u_{n}^{\prime}(t)=\lim _{t \rightarrow 0^{+}} w(t) u^{\prime}(t) .
\end{aligned}
$$

Thus $u \in C_{w}[0,1]$ and $w u^{\prime} \equiv v$ on $[0,1]$. This implies $u_{n} \rightarrow u$ in $C_{w}[0,1]$ and the proof is completed.

\section{Define}

$$
X=\left\{u \in C_{w}[0,1] \mid u(0)=0=u(1)\right\} .
$$

Then we see that for given $h \in \mathscr{H} \backslash L^{1}(0,1), u$ being a solution of $(P)$ implies $u \in X$. In fact, if $\lim _{t \rightarrow 0^{+}} \int_{t}^{1 / 2} h(s) d s=\infty$ and $u$ is a solution of $(P)$, then for $t \in(0,1 / 2)$,

$$
u^{\prime}(t)=\varphi_{p}^{-1}\left(\int_{t}^{1 / 2} h(s) f(u(s)) d s+\varphi_{p}\left(u^{\prime}\left(\frac{1}{2}\right)\right)\right),
$$

and by using L'Hospital's rule, we have

$$
\begin{aligned}
& \lim _{t \rightarrow 0^{+}} w(t) u^{\prime}(t) \\
& =\lim _{t \rightarrow 0^{+}} \varphi_{p}^{-1}\left(\frac{\int_{t}^{1 / 2} h(s) f(u(s)) d s+\varphi_{p}\left(u^{\prime}(1 / 2)\right)}{\int_{t}^{1 / 2} h(s) d s}\right) \\
& =\lim _{t \rightarrow 0^{+}} \varphi_{p}^{-1}\left(\lim _{t \rightarrow 0^{+}} \frac{h(t) f(u(t))}{h(t)}\right)=\varphi_{p}^{-1}(f(0)) .
\end{aligned}
$$


For the cases that $\lim _{t \rightarrow 0^{+}} \int_{t}^{1 / 2} h(s) d s=-\infty$ and $\lim _{t \rightarrow 1^{-}}$ $\int_{1 / 2}^{t} h(s) d s=\infty($ or $-\infty)$, by the same argument, we have

$$
\begin{aligned}
& -\infty<\lim _{t \rightarrow 0^{+}} w(t) u^{\prime}(t)<\infty, \\
& -\infty<\lim _{t \rightarrow 1^{-}} w(t) u^{\prime}(t)<\infty .
\end{aligned}
$$

Example 2. Let us consider the following example:

$$
\begin{aligned}
\varphi_{p}\left(u^{\prime}(t)\right)^{\prime}+h(t) \exp \left(\frac{\alpha u}{\alpha+u}\right) & =0, \quad t \in(0,1], \\
u(0)=0, \quad u(1) & =0
\end{aligned}
$$

where $\alpha, \lambda>0, p>3 / 2$ and $h:(0,1) \rightarrow \mathbb{R}$ is given by

$$
h(t)= \begin{cases}t^{-3 / 2}, & 0<t \leq \frac{1}{2} \\ -1, & \frac{1}{2}<t<1\end{cases}
$$

Then we see that $h \in \mathscr{H} \backslash L^{1}(0,1]$ sign-changing and every solution $u$ satisfies $u^{\prime}\left(0^{+}\right)=\infty$ by using (9). We also see by calculation that $w$ can be given as

$$
w(t)= \begin{cases}\left(\frac{\sqrt{t}}{2(1-\sqrt{2 t})}\right)^{1 /(p-1)}, & \text { if } t \in\left(0, \frac{36-16 \sqrt{2}}{49}\right], \\ 1, & \text { if } t \in\left(\frac{36-16 \sqrt{2}}{49}, 1\right],\end{cases}
$$

and $w u^{\prime}\left(0^{+}\right)=1$ by using $(10)$.

To establish corresponding integral operator for problem $(P)$, let us first consider the problem

$$
\begin{gathered}
-\varphi_{p}\left(u^{\prime}\right)^{\prime}=g, \quad t \in(0,1), \\
u(0)=0=u(1),
\end{gathered}
$$

where $g \in \mathscr{H}$. We remind the reader that $g$ needs not be integrable near $t=0$ or 1 . Integrating on $[t, 1 / 2]$ for $t \epsilon$ $(0,1 / 2]$, we have

$$
\varphi_{p}\left(u^{\prime}(t)\right)=\varphi_{p}\left(u^{\prime}\left(\frac{1}{2}\right)\right)+\int_{t}^{1 / 2} g(r) d r
$$

Denoting $\rho=\varphi_{p}\left(u^{\prime}(1 / 2)\right)$,

$$
u^{\prime}(t)=\varphi_{p}^{-1}\left(\rho+\int_{t}^{1 / 2} g(r) d r\right) .
$$

Since $g \in \mathscr{H}$ and $\rho$ is a fixed constant, we can see that

$$
\varphi_{p}^{-1}\left(\rho+\int_{t}^{1 / 2} g(r) d r\right) \in L^{1}\left(0, \frac{1}{2}\right)
$$

so that we may integrate on $(0, t)$. Using a boundary condition $u(0)=0$, we get

$$
u(t)=\int_{0}^{t} \varphi_{p}^{-1}\left(\rho+\int_{t}^{1 / 2} g(r) d r\right) d s, \quad \text { for } t \in\left[0, \frac{1}{2}\right] .
$$

We note that $u$ in (18) is a solution of $(A)$ only on the interval $[0,1 / 2]$. Doing similar computation on the interval $[1 / 2,1]$, we get

$$
u(t)=\int_{t}^{1} \varphi_{p}^{-1}\left(-\rho+\int_{1 / 2}^{t} g(r) d r\right) d s, \quad \text { for } t \in\left[\frac{1}{2}, 1\right) .
$$

It is known by Lemma 2.2 in [10] that the equation

$$
\begin{aligned}
& \int_{0}^{1 / 2} \varphi_{p}^{-1}\left(\rho+\int_{s}^{1 / 2} g(r) d r\right) d s \\
& \quad=\int_{1 / 2}^{1} \varphi_{p}^{-1}\left(-\rho+\int_{1 / 2}^{s} g(r) d r\right) d s
\end{aligned}
$$

has a unique zero $\rho \triangleq \rho(g)$ in $\mathbb{R}$ for each $g \in \mathscr{H}$. Therefore it is natural to paste $u$ 's in (18) and (19) in a continuous way. Now let us define a function $u$ by

$$
u(t)= \begin{cases}\int_{0}^{t} \varphi_{p}^{-1}\left(\rho(g)+\int_{s}^{1 / 2} g(r) d r\right) d s, & 0 \leq t \leq \frac{1}{2} \\ \int_{t}^{1} \varphi_{p}^{-1}\left(-\rho(g)+\int_{1 / 2}^{s} g(r) d r\right) d s, & \frac{1}{2} \leq t \leq 1\end{cases}
$$

Then $u$ satisfies $u \in C[0,1] \cap C^{1}(0,1)$ and $u$ is a unique solution of problem $(A)$.

Based on this setup, we now introduce corresponding integral operator for problem $(P)$. For $u \in C[0,1]$, define

$$
\begin{aligned}
& T u(t) \\
& =\left\{\begin{array}{l}
\int_{0}^{t} \varphi_{p}^{-1}(\rho(h f(u)) \\
\left.\quad+\int_{s}^{1 / 2} h(\tau) f(u(\tau)) d \tau\right) d s, \quad 0 \leq t \leq \frac{1}{2}, \\
\int_{t}^{1} \varphi_{p}^{-1}(-\rho(h f(u)) \\
\left.\quad+\int_{1 / 2}^{s} h(\tau) f(u(\tau)) d \tau\right) d s, \quad \frac{1}{2} \leq t \leq 1 .
\end{array}\right.
\end{aligned}
$$

Then $u=T u$ in $C[0,1]$ if and only if $u$ is a solution of $(P)$.

Remark 3. We understand the number $\rho(h f(u))$ in the above as a function of $u$ defined on $C[0,1]$. That is, $\rho: C[0,1] \rightarrow$ $\mathbb{R}$. It is known that $\rho$ maps bounded sets in $C[0,1]$ into bounded sets in $\mathbb{R}([10$, Lemma 3.1$])$. It is also known that $T$ is completely continuous on $C[0,1]$ ([10, Theorem 3.4$])$.

As mentioned in Introduction, the regularity of solutions of problem $(P)$ sensitively depends on the shape of nonlinear 
term $f$ even if $h \in \mathscr{H} \backslash L^{1}(0,1)$, and we are concerned with the case that problem $(P)$ does not have $C^{1}$-solutions. Therefore, it is interesting to consider operator $T$ restricted on $X$ to complete three solutions theorem for those problems with no $C^{1}$-solutions.

Define $G$ the restriction of $T$ on $X$.

In what is to follow, we assume $h \in \mathscr{H} \backslash L^{1}(0,1)$ and we now prove the complete continuity of $G$ on the solution space $X$. Before doing that, we give a remark useful to calculate p-Laplacians.

Remark 4. If $a, b>0$, then

$$
\varphi_{p}^{-1}(a+b) \leq C_{p}\left(\varphi_{p}^{-1}(a)+\varphi_{p}^{-1}(b)\right),
$$

where

$$
C_{p}:= \begin{cases}1, & p>2, \\ 2^{(2-p) /(p-1)}, & 1<p \leq 2 .\end{cases}
$$

Theorem 5. $G: X \rightarrow X$ is completely continuous.

Proof. Let $B$ be a bounded subset of $X$. Then for any sequence $\left(u_{n}\right) \subset B$, we need to show the relative compactness of $\left(G u_{n}\right)$ with respect to $\|\cdot\|_{w}$-norm. We know by Remark 3 that $G$ is completely continuous on $C[0,1]$ so that there exists $u_{0} \in$ $C[0,1]$ and a subsequence of $\left(u_{n}\right)$, say again $\left(u_{n}\right)$ such that $G u_{n} \rightarrow u_{0}$ in $C[0,1]$. To complete the proof, we need to show the following.

$u_{0} \in X$ and there is a subsequence $\left(G u_{n l}\right)$ of $\left(G u_{n}\right)$ such that $G u_{n l} \rightarrow u_{0}$ as $l \rightarrow \infty$ in $X$ and $G$ is continuous on $X$.

Claim 1. $\left\{w\left(G u_{n}\right)^{\prime}\right\}$ is uniform bounded in $C[0,1]$.

Since $B$ is bounded in $X$, there exists $M_{B}>0$ such that $\|u\|_{\infty}<M_{B}$ and $\left\|w u^{\prime}\right\|_{\infty}<M_{B}$, for all $u \in B$. We also know by Remark 3 that there is $N_{B}>0$ such that $|\rho(h f(u))|<N_{B}$, for all $u \in B$. For $t \in(0,1 / 2)$, by using Remark 4 , we get

$$
\begin{aligned}
\left|\left(G u_{n}\right)^{\prime}(t)\right| & \leq \varphi_{p}^{-1}\left(|\rho(h f(u))|+\int_{t}^{1 / 2}|h(s)|\left|f\left(u_{n}(s)\right)\right| d s\right) \\
& \leq \varphi_{p}^{-1}\left(N_{B}+\bar{f} \int_{t}^{1 / 2}|h(s)| d s\right) \\
& \leq C_{p}\left(\varphi_{p}^{-1}\left(N_{B}\right)+\varphi_{p}^{-1}(\bar{f}) \varphi_{p}^{-1}\left(\int_{t}^{1 / 2}|h(s)| d s\right)\right),
\end{aligned}
$$

where $\bar{f}=\max _{s \in\left[-M_{B}, M_{B}\right]}|f(s)|$. From the fact that $0 \leq w(t) \leq$ 1 and the definition of $w(t)$, we see

$$
w(t) \varphi_{p}^{-1}\left(\int_{t}^{1 / 2}|h(s)| d s\right) \leq 1,
$$

thus we have

$$
w(t)\left|\left(G u_{n}\right)^{\prime}(t)\right| \leq C_{p}\left(\varphi_{p}^{-1}\left(N_{B}\right)+\varphi_{p}^{-1}(\bar{f})\right),
$$

for $t \in(0,1 / 2]$. For $t \in[1 / 2,1)$, by similar calculation, we get the same upper bound of $w(t)\left|\left(G u_{n}\right)^{\prime}(t)\right|$ as in (27) and by taking limt $t \rightarrow 0^{+}$and $t \rightarrow 1^{-}$in (27), we have the same upper bound of $\left|w\left(G u_{n}\right)^{\prime}(0)\right|$ and $\left|w\left(G u_{n}\right)^{\prime}(1)\right|$ as in (27). This proves that $\left\{w\left(G u_{n}\right)^{\prime}\right\}$ is bounded in $C[0,1]$.

Claim 2. $\left\{w\left(G u_{n}\right)^{\prime}\right\}$ is equicontinuous on $[0,1]$.

If $h \in L^{1}(0,1)$, then since $\left\|w u_{n}^{\prime}\right\|_{\infty}<M_{B}$, for all $n$ and $w^{-1} \in L^{1}(0,1)$, we get

$$
\left|u_{n}^{\prime}\right|<M_{B}(w)^{-1} \in L^{1}(0,1),
$$

for all $n$. This implies that $\left\{u_{n}\right\}$ is equicontinuous in $C[0,1]$ and by Arzela-Ascoli theorem, there exist a subsequence $\left\{u_{n k}\right\}$ of $\left\{u_{n}\right\}$ and $v \in C[0,1]$ such that $u_{n k}$ converges uniformly to $v$ on $[0,1]$ as $k \rightarrow \infty$. Thus using Lebesgue Dominated Convergence theorem, we obtain

$$
\begin{aligned}
& w(t) \varphi_{p}^{-1}\left(\rho\left(h f\left(u_{n k}\right)\right)+\int_{t}^{1 / 2} h(s) f\left(u_{n k}(s)\right) d s\right) \\
& \longrightarrow w(t) \varphi_{p}^{-1}\left(\rho(h f(v))+\int_{t}^{1 / 2} h(s) f(v(s)) d s\right),
\end{aligned}
$$

uniformly on $[0,1]$. This implies that $\left\{w\left(G u_{n}\right)^{\prime}\right\}$ is equicontinuous in $C[0,1]$.

On the other hand, for the case of $h \in \mathscr{H} \backslash L^{1}(0,1)$, suppose that $\left\{w\left(G u_{n}\right)^{\prime}\right\}$ is not equicontinuous on $[0,1]$. Then there exists $\varepsilon>0$ such that we may choose a subsequence $\left\{u_{n k}\right\}$ of $\left\{u_{n}\right\}$ and sequences $\left\{t_{k}\right\},\left\{s_{k}\right\} \subset(0,1)$ satisfying

$$
\begin{gathered}
\left|t_{k}-s_{k}\right|<\frac{1}{k}, \\
\left|w\left(G u_{n k}\right)^{\prime}\left(t_{k}\right)-w\left(G u_{n k}\right)^{\prime}\left(s_{k}\right)\right| \geq \varepsilon .
\end{gathered}
$$

As for sequences $\left\{t_{k}\right\}$ and $\left\{s_{k}\right\}$, it is easy to see that $\lim _{k \rightarrow \infty} t_{k}=\lim _{k \rightarrow \infty} s_{k}$. We show that $\lim _{k \rightarrow \infty} t_{k}=0$ or 1. Suppose it is not true so let $\lim _{k \rightarrow \infty} t_{k}=t_{0} \in(0,1)$. Then taking $\eta$ satisfying $0<\eta<\min \left\{t_{0}, 1-t_{0}\right\}$, we see that $h \in L^{1}[\eta, 1-\eta]$ and $u_{n k} \rightarrow v$ uniformly on $[\eta, 1-\eta]$. By the same argument of the above case of $h \in L^{1}(0,1)$, we can prove that $\left\{w\left(G u_{n}\right)^{\prime}\right\}$ is equicontinuous on $[\eta, 1-\eta]$. Thus there is sufficiently large $N \in \mathbb{N}$ such that

$$
\left|w\left(G u_{n N}\right)^{\prime}\left(t_{N}\right)-w\left(G u_{n N}\right)^{\prime}\left(s_{N}\right)\right|<\varepsilon,
$$

and this contradicts with (30). Now we consider the case $\lim _{k \rightarrow \infty} t_{k}=0=\lim _{k \rightarrow \infty} s_{k}$. The argument for the case $\lim _{k \rightarrow \infty} t_{k}=1=\lim _{k \rightarrow \infty} s_{k}$ is similar. It is easy to see that this case implies $\lim _{k \rightarrow \infty} w\left(t_{k}\right)=0$. Since $w\left(t_{k}\right) \geq 0$, $w\left(t_{k}\right)=\varphi_{p}^{-1}\left(w^{p-1}\left(t_{k}\right)\right)$, for all $k$ and we get

$$
\begin{aligned}
& w\left(G u_{n k}\right)^{\prime}\left(t_{k}\right) \\
& =\varphi_{p}^{-1}\left(w^{p-1}\left(t_{k}\right) \rho\left(h f\left(u_{n k}\right)\right)\right. \\
& \left.\quad+w^{p-1}\left(t_{k}\right) \int_{t_{k}}^{1 / 2} h(s) f\left(u_{n k}(s)\right) d s\right) .
\end{aligned}
$$

Now we want to calculate $\lim _{k \rightarrow \infty} w\left(G u_{n k}\right)^{\prime}\left(t_{k}\right)$. 

have

Since $\rho\left(h f\left(u_{n k}\right)\right)$ is bounded and $\lim _{k \rightarrow \infty} w\left(t_{k}\right)=0$, we

$$
\lim _{k \rightarrow \infty} w^{p-1}\left(t_{k}\right) \rho\left(h f\left(u_{n k}\right)\right)=0 .
$$

On the other hand,

$$
\begin{aligned}
w^{p-1}\left(t_{k}\right) \int_{t_{k}}^{1 / 2} h(s) f\left(u_{n k}(s)\right) d s \\
=w^{p-1}\left(t_{k}\right) \int_{t_{k}}^{1 / 2} h(s)\left[f\left(u_{n k}(s)\right)-f(v(s))\right] d s \\
\quad+w^{p-1}\left(t_{k}\right) \int_{t_{k}}^{1 / 2} h(s) f(v(s)) d s .
\end{aligned}
$$

We show $\lim _{k \rightarrow \infty} w^{p-1}\left(t_{k}\right) \int_{t_{k}}^{1 / 2} h(s)\left[f\left(u_{n k}(s)\right)-f(v(s))\right] d s=$ 0 .

Indeed, $w^{p-1}\left(t_{k}\right)$ is close to 0 for sufficiently large $k$, since $p-1>0$ and $\lim _{k \rightarrow \infty} w\left(t_{k}\right)=0$. Therefore, without loss of generality, we may assume that $w\left(t_{k}\right)=\left(\varphi_{p}^{-1}\left(\int_{t_{k}}^{1 / 2}|h(s)| d s\right)\right)^{-1}$ so that $w^{p-1}\left(t_{k}\right) \int_{t_{k}}^{1 / 2}|h(s)| d s=$ $\left(\int_{t_{k}}^{1 / 2}|h(s)| d s\right)^{-1} \int_{t_{k}}^{1 / 2}|h(s)| d s=1$. Thus using the fact $u_{n k} \rightarrow$ $v$ in $C[0,1]$, we have

$$
\begin{aligned}
& \lim _{k \rightarrow \infty}\left|w^{p-1}\left(t_{k}\right) \int_{t_{k}}^{1 / 2} h(s)\left[f\left(u_{n k}(s)\right)-f(v(s))\right] d s\right| \\
& \quad \leq \lim _{k \rightarrow \infty} w^{p-1}\left(t_{k}\right) \int_{t_{k}}^{1 / 2}|h(s)| d s\left\|f\left(u_{n k}\right)-f(v)\right\|_{\infty} \\
& \quad=\lim _{k \rightarrow \infty}\left\|f\left(u_{n k}\right)-f(v)\right\|_{\infty}=0 .
\end{aligned}
$$

Next we show

$$
\begin{gathered}
\lim _{k \rightarrow \infty} w^{p-1}\left(t_{k}\right) \int_{t_{k}}^{1 / 2} h(s) f(v(s)) d s \\
\quad= \begin{cases}f(0), & \text { if } h>0 \text { near } 0, \\
-f(0), & \text { if } h<0 \text { near } 0 .\end{cases}
\end{gathered}
$$

Indeed, using the fact $w^{p-1}\left(t_{k}\right)=\left(\int_{t_{k}}^{1 / 2}|h(s)| d s\right)^{-1}$ for sufficiently large $k$, we get

$$
\begin{gathered}
\lim _{k \rightarrow \infty} w^{p-1}\left(t_{k}\right) \int_{t_{k}}^{1 / 2} h(s) f(v(s)) d s \\
=\lim _{t \rightarrow 0^{+}} \frac{\int_{t}^{1 / 2} h(s) f(v(s)) d s}{\int_{t}^{1 / 2}|h(s)| d s} .
\end{gathered}
$$

If $h f(v) \in L^{1}(0,1 / 2)$, then we can easily verify $f(0)=0$. Since $\lim _{t \rightarrow 0^{+}} \int_{t}^{1 / 2}|h(s)| d s=\infty$, we see that the limit is 0 . On the other hand, if $h f(v) \notin L^{1}(0,1 / 2)$. We note that $h \in$ $L^{1}[t, 1 / 2]$, for given $t>0$. By using L'Hospital's rule, we get the conclusion.
Therefore we get

$$
\lim _{k \rightarrow \infty} w\left(G u_{n k}\right)^{\prime}\left(t_{k}\right)= \begin{cases}f(0), & \text { if } h>0 \text { near } 0, \\ -f(0), & \text { if } h<0 \text { near } 0 .\end{cases}
$$

By the same argument, $w\left(G u_{n k}\right)^{\prime}\left(s_{k}\right)$ also has the same limit and this contradicts with (30). Consequently, $\left\{w\left(G u_{n}\right)^{\prime}\right\}$ is equicontinuous in $C[0,1]$. By Arzela-Ascoli theorem, there exists a subsequence $\left\{u_{n l}\right\}$ of $\left\{u_{n}\right\}$ and $z \in C[0,1]$ such that

$$
w\left(G u_{n l}\right)^{\prime} \longrightarrow z \quad \text { as } l \longrightarrow \infty, \quad \text { in } C[0,1] .
$$

Claim 3. $u_{0} \in X$ and $G u_{n l} \rightarrow u_{0}$ in $X$.

It is enough to show that $z \equiv w u_{0}^{\prime}$. Since $w(t)>0$ for $t \in(0,1)$, let $r(t):=z(t) / w(t)$; then $r \in C((0,1))$ and for $\delta>0,\left(G u_{n l}\right)^{\prime} \rightarrow r$ uniformly in $C[\delta, 1-\delta]$ and thus $u_{0}^{\prime} \equiv r$ on $[\delta, 1-\delta]$. Since $\delta$ is arbitrary, $u_{0}^{\prime} \equiv r$ on $(0,1)$ and from (39), we have

$$
\begin{aligned}
z(0) & =\lim _{l \rightarrow \infty} w\left(G u_{n l}\right)^{\prime}(0)=\lim _{l \rightarrow \infty} \lim _{t \rightarrow 0} w(t)\left(G u_{n l}\right)^{\prime}(t) \\
& =\lim _{t \rightarrow 0} \lim _{l \rightarrow \infty} w(t)\left(G u_{n l}\right)^{\prime}(t)=\lim _{t \rightarrow 0} w(t) u_{0}^{\prime}(t) .
\end{aligned}
$$

Therefore $w u_{0}^{\prime} \equiv z$ on $[0,1)$. By similar argument near 1 , we get $w u^{\prime} \equiv z$ on $[0,1]$ and thus $u_{0} \in X$.

Claim 4. $G$ is continuous on $X$.

Assume that $u_{n} \rightarrow \widetilde{u}$ in X. By compactness of $G$, there is a subsequence $\left\{u_{n j}\right\}$ of $\left\{u_{n}\right\}$ and $v \in X$ such that $G\left(u_{n j}\right) \rightarrow v$ in $X$. It is suffice to see that $G \tilde{u}(t)=v(t)$, for all $t \in[0,1]$. Since $G$ is continuous in $C[0,1]$ and $u_{n j} \rightarrow \widetilde{u}$ in $C[0,1]$, we have $G u_{n j} \rightarrow G \tilde{u}$ in $C[0,1]$. Thus $G \tilde{u} \equiv v$ and by standard limit argument, we see that $G\left(u_{n}\right) \rightarrow v=G \tilde{u}$. This completes the proof.

Now we define a strong sense of ordering in $C_{w}[0,1]$.

Definition 6. For $u, v \in C_{w}[0,1]$, one says that $u \prec v$ if and only if

(i) $u(t)<v(t)$ for all $t \in(0,1)$,

(ii) either $u(0)<v(0)$ or $w u^{\prime}(0)<w v^{\prime}(0)$,

(iii) either $u(1)<v(1)$ or $w u^{\prime}(1)>w v^{\prime}(1)$.

Definition 7. One says that $\alpha$ is a lower solution of $(P)$ if and only if $\alpha \in C_{w}[0,1], \varphi_{p}\left(\alpha^{\prime}\right) \in C^{1}(0,1)$ and

$$
\begin{gathered}
\varphi_{p}\left(\alpha^{\prime}(t)\right)^{\prime}+f(t, \alpha(t)) \geq 0, \quad t \in(0,1), \\
\alpha(0) \leq 0, \quad \alpha(1) \leq 0 .
\end{gathered}
$$

We also say that $\beta$ is an upper solution of $(P)$ if and only if $\beta \epsilon$ $C_{w}[0,1]$ with $\varphi_{p}\left(\beta^{\prime}\right) \in C^{1}(0,1)$ and it satisfies the reverse of the above inequalities.

Definition 8. One says that $\alpha$ is a strict lower solution of $(P)$ if and only if $\alpha$ is a lower solution of $(P)$ and satisfies $\alpha<u$ where $u$ is a solution of $(P)$ such that $u(t) \geq \alpha(t), t \in[0,1]$.

We say that $\beta$ is a strict upper solution of $(P)$ if and only if $\beta$ is an upper solution of $(P)$ and satisfies $u<\beta$ where $u$ is a solution of $(P)$ such that $\beta(t) \geq u(t), t \in[0,1]$. 
Theorem 9. Assume that there exist a strict lower solution $\alpha$ and a strict upper solution $\beta$ of $(P)$ such that $\alpha \prec \beta$. Then problem $(P)$ has at least one solution $u$ such that $\alpha \prec u \prec \beta$. Moreover, for $R>0$ large enough, the Leray-Schauder degree can be computed as

$$
d_{L S}(I-G, \Omega, 0)=1,
$$

where $\Omega=\left\{u \in X \mid \alpha \prec u \prec \beta,\|u\|_{w}<R\right\}$.

Proof. Consider the modified problem

$$
\begin{gathered}
\varphi_{p}\left(u^{\prime}(t)\right)^{\prime}+h(t) f(\gamma(t, u(t)))=0, \quad t \in(0,1), \\
u(0)=0, \quad u(1)=0,
\end{gathered}
$$

where $\gamma:(0,1) \times \mathbb{R} \rightarrow \mathbb{R}$ is defined by

$$
\gamma(t, u)= \begin{cases}\beta(t), & u>\beta(t), \\ u, & \alpha(t) \leq u \leq \beta(t), \\ \alpha(t), & u<\alpha(t) .\end{cases}
$$

Then it is well known that if $u$ is a solution of $(M)$, then $\alpha(t) \leq$ $u(t) \leq \beta(t)$ and thus $u$ is solution of $(P)$. Define $\bar{G}: X \rightarrow X$ by $\bar{G}(u)(t)=G(\gamma(t, u(t)))$. Then $\bar{G}$ is bounded and thus there exists $R \gg 1$ such that $\|\bar{G} u\|_{w}<R$ for all $u \in X$. By the homotopy invariance property of degree, we have

$$
\mathrm{d}_{\mathrm{LS}}\left(I-\bar{G}, B_{R}(0), 0\right)=\mathrm{d}_{\mathrm{LS}}\left(I, B_{R}(0), 0\right)=1,
$$

where $B_{R}(0)=\left\{u \in X \mid\|u\|_{w}<R\right\}$. Thus $(M)$ has a solution and $(P)$ has a solution $u$ satisfying $\alpha(t) \leq u(t) \leq \beta(t)$. Since $\alpha$ and $\beta$ are strict lower and upper solutions, respectively, by the definition of strict lower and upper solution, we have $\alpha \prec$ $u \prec \beta$. Moreover, by using the fact that $\bar{G}=G$ on $\bar{\Omega},(44)$ and excision property, we conclude that there exists $R>0$ large enough such that

$$
\begin{aligned}
\mathrm{d}_{\mathrm{LS}}(I-G, \Omega, 0) & =\mathrm{d}_{\mathrm{LS}}(I-\bar{G}, \Omega, 0) \\
& =\mathrm{d}_{\mathrm{LS}}\left(I-\bar{G}, B_{R}(0), 0\right)=1 .
\end{aligned}
$$

Theorem 10 (three solutions theorem). Assume that there exist a lower solution $\alpha_{1}$, an upper solution $\beta_{2}$, a strict lower solution $\alpha_{2}$, and a strict upper solution $\beta_{1}$ of $(P)$ such that

$$
\alpha_{1} \leq \beta_{1} \leq \beta_{2}, \quad \alpha_{1} \leq \alpha_{2} \leq \beta_{2},
$$

and there exists $t_{0} \in[0,1]$ with $\beta_{1}\left(t_{0}\right)<\alpha_{2}\left(t_{0}\right)$. Then problem $(P)$ has at least three solutions $u_{1}, u_{2}$, and $u_{3}$ such that

$$
\begin{gathered}
\alpha_{1} \leq u_{1} \prec \beta_{1}, \quad \alpha_{2} \prec u_{2} \leq \beta_{2}, \\
u_{3} \in\left[\alpha_{1}, \beta_{2}\right] \backslash\left(\left[\alpha_{1}, \beta_{1}\right] \cup\left[\alpha_{2}, \beta_{2}\right]\right) .
\end{gathered}
$$

Proof. Consider the modified problem,

$$
\begin{gathered}
\varphi_{p}\left(u^{\prime}(t)\right)^{\prime}+h(t) f\left(\gamma_{1,2}(t, u(t))\right)=0, \quad t \in(0,1), \\
u(0)=0, \quad u(1)=0,
\end{gathered}
$$

where $\gamma_{1,2}:(0,1) \times \mathbb{R} \rightarrow \mathbb{R}$ is defined by

$$
\gamma_{1,2}(t, u)= \begin{cases}\beta_{2}(t), & u>\beta_{2}(t) \\ u, & \alpha_{1}(t) \leq u \leq \beta_{2}(t) \\ \alpha_{1}(t), & u<\alpha_{1}(t)\end{cases}
$$

For any $\varepsilon>0, \alpha_{1}-\varepsilon$ and $\beta_{2}+\varepsilon$ are strict lower solution and strict upper solution of $\left(M_{1,2}\right)$. In fact, if $u$ is a solution of $\left(M_{1,2}\right)$, then we have $\alpha_{1}(t)-\varepsilon<\alpha_{1}(t) \leq u(t) \leq \beta_{2}(t)<$ $\beta_{2}(t)+\varepsilon$. By Theorem 5, there is a sufficient large $R>0$ :

$$
\begin{aligned}
& \mathrm{d}_{\mathrm{LS}}\left(I-G_{1,2}, \Omega_{1,1}, 0\right)=1, \\
& \mathrm{~d}_{\mathrm{LS}}\left(I-G_{1,2}, \Omega_{2,2}, 0\right)=1, \\
& \mathrm{~d}_{\mathrm{LS}}\left(I-G_{1,2}, \Omega_{1,2}, 0\right)=1,
\end{aligned}
$$

where $G_{1,2}: X \rightarrow X$ is defined by $G_{1,2}(u)(t)=G\left(\gamma_{1,2}(t, u(t))\right.$ and

$$
\begin{aligned}
& \Omega_{1,1}=\left\{u \in X \alpha_{1}-\varepsilon \prec u \prec \beta_{1},\|u\|_{w}<R\right\}, \\
& \Omega_{2,2}=\left\{u \in X \alpha_{2} \prec u \prec \beta_{2}+\varepsilon,\|u\|_{w}<R\right\}, \\
& \Omega_{1,2}=\left\{u \in X \mid \alpha_{1}-\varepsilon \prec u \prec \beta_{2}+\varepsilon,\|u\|_{w}<R\right\} .
\end{aligned}
$$

Then by excision and additive property, we have

$$
\mathrm{d}_{\mathrm{LS}}\left(I-G_{1,2}, \Omega_{1,2} \backslash\left(\bar{\Omega}_{1,1} \cup \bar{\Omega}_{2,2}\right), 0\right)=-1 .
$$

Thus there are three solutions of $\left(M_{1,2}\right), u_{1} \in \bar{\Omega}_{1,1}, u_{2} \in \bar{\Omega}_{2,2}$, and $u_{3} \in \Omega_{1,2} \backslash\left(\bar{\Omega}_{1,1} \cup \bar{\Omega}_{2,2}\right)$. Since all solutions $u$ of $\left(M_{1,2}\right)$ satisfy $u \in\left[\alpha_{1}, \beta_{2}\right]$, they are solutions of $(P)$ and the proof is done.

\section{Application}

In this section, we prove the existence of triple positive solutions for a problem of the form

$$
\begin{gathered}
\varphi_{p}\left(u^{\prime}(t)\right)^{\prime}+\lambda h(t) f(u(t))=0, \quad t \in(0,1), \\
u(0)=0=u(1)
\end{gathered}
$$

where $\lambda>0$ and $f \in C([0, \infty),(0, \infty))$. Let us assume

$$
\begin{aligned}
& \left(H_{1}\right) h \in \mathscr{H} \backslash L^{1}(0,1) \text { with } h \geq 0, \\
& \left(H_{2}\right) \min _{t \in(0,1)} h(t)=\underline{h}>0, \\
& \left(F_{1}\right) \lim _{u \rightarrow \infty}\left(f(u) / \varphi_{p}(u)\right)=0, \\
& \left(F_{2}\right) f \text { is nondecreasing. }
\end{aligned}
$$

The existence of two positive solutions for problem $\left(P_{\lambda}\right)$ was proved in [11] under a stronger condition on $h$ such as $\int_{0}^{1} s^{\delta}(1-s)^{\gamma} h(s) d s<\infty$ for some $\delta, \gamma<p-1$. Since $f(0)>0$, we can easily see that any solution $u$ of problem $\left(P_{\lambda}\right)$ is in $C_{0}[0,1]$ but not in $C^{1}[0,1]$ so that with the aid of three solutions theorem in Section 2, we prove the following theorem. 
Theorem 11. Assume $\left(H_{1}\right),\left(H_{2}\right),\left(F_{1}\right)$ and $\left(F_{2}\right)$, and also assume that there exist $a>0$ and $b>0$ such that $a<b$ and

$$
\frac{a^{p-1}}{f(a)}>C \frac{b^{p-1}}{f(b)},
$$

where $C=4^{p}\left(\|e\|_{\infty}^{p-1} / \underline{h}\right)$ and e the unique solution of

$$
\begin{gathered}
\varphi_{p}\left(e^{\prime}(t)\right)^{\prime}+h(t)=0, \quad t \in(0,1) \\
e(0)=0=e(1) .
\end{gathered}
$$

Then for $\lambda>0$ satisfying

$$
\frac{b^{p-1} C}{f(b)\|e\|_{\infty}^{p-1}}<\lambda<\frac{a^{p-1}}{f(a)\|e\|_{\infty}^{p-1}},
$$

problem $\left(P_{\lambda}\right)$ has at least three positive solutions.

Proof. For $\lambda \in\left(b^{p-1} C / f(b)\|e\|_{\infty}^{p-1}, a^{p-1} / f(a)\|e\|_{\infty}^{p-1}\right)$, it is trivial that $\alpha_{1} \equiv 0$ is a lower solution of $\left(P_{\lambda}\right)$. Let $\beta_{1}=$ $a\left(e /\|e\|_{\infty}\right)$. Then

$$
\begin{aligned}
-\varphi_{p}\left(\beta_{1}^{\prime}(t)\right)^{\prime} & =-\frac{a^{p-1}}{\|e\|_{\infty}^{p-1}} \varphi_{p}\left(e^{\prime}(t)\right)^{\prime}=\frac{a^{p-1}}{\|e\|_{\infty}^{p-1}} h(t) \\
& >\lambda h(t) f(a) \geq \lambda h(t) f\left(\beta_{1}(t)\right) .
\end{aligned}
$$

Thus $\beta_{1}$ is an upper solution of $\left(P_{\lambda}\right)$. To show that $\beta_{1}$ is a strict upper solution of $\left(P_{\lambda}\right)$, assume that $u$ is a solution of $\left(P_{\lambda}\right)$ such that $u \leq \beta_{1}$. We first show that $u(t)<\beta_{1}(t)$, for all $t \in(0,1)$. Suppose it is not true, then there exist $t_{0}$ and $t_{1}$ with $t_{0}<t_{1}$ in $(0,1)$ such that $u^{\prime}\left(t_{0}\right)=\beta_{1}^{\prime}\left(t_{0}\right)$ and $u^{\prime}\left(t_{1}\right)<\beta_{1}^{\prime}\left(t_{1}\right)$. Integrate $\varphi_{p}\left(u^{\prime}(t)\right)^{\prime}-\varphi_{p}\left(\beta_{1}^{\prime}(t)\right)^{\prime}$ from $t_{0}$ to $t_{1}$, by $(55)$ and monotonicity of $f$, we have the following contradiction:

$$
\begin{aligned}
0 & >\varphi_{p}\left(u^{\prime}\left(t_{1}\right)\right)-\varphi_{p}\left(\beta_{1}^{\prime}\left(t_{1}\right)\right) \\
& =\int_{t_{0}}^{t_{1}} \varphi_{p}\left(u^{\prime}(s)\right)^{\prime}-\varphi_{p}\left(\beta_{1}^{\prime}(s)\right)^{\prime} d s \\
& >\int_{t_{0}}^{t_{1}}-\lambda h(s) f(u(s))+\lambda h(s) f\left(\beta_{1}(s)\right) \geq 0 .
\end{aligned}
$$

Since $u(0)=\beta_{1}(0)=u(1)=\beta_{1}(1)=0$, it suffices to show that $w u^{\prime}(1)>w \beta_{1}^{\prime}(1)$. The inequality $w u^{\prime}(0)<w \beta_{1}^{\prime}(0)$ can be proved similarly. For the case that $\lim _{t \rightarrow 1^{-}} \int_{1 / 2}^{t} h(s) d s<\infty$, we know $u^{\prime}(1)$ and $\beta^{\prime}(1)$ exist. Since

$$
\varphi_{p}\left(u^{\prime}(t)\right)^{\prime}-\varphi_{p}\left(\beta_{1}^{\prime}(t)\right)^{\prime}>0, \quad t \in(0,1),
$$

we know that there exists $d \in(0,1)$ such that $u^{\prime}(d)>\beta^{\prime}(d)$. Indeed, otherwise, $u^{\prime}(t) \leq \beta^{\prime}(t)$ for all $t \in(0,1)$; then by integrating this from $t$ to 1 , we have the contradiction

$$
u(t) \geq \beta(t), \quad t \in(0,1) .
$$

Integrating (57) from $d$ to 1 , we have

$$
\varphi_{p}\left(u^{\prime}(1)\right)-\varphi_{p}\left(\beta_{1}^{\prime}(1)\right)>\varphi_{p}\left(u^{\prime}(d)\right)-\varphi_{p}\left(\beta_{1}^{\prime}(d)\right)>0,
$$

and thus $u^{\prime}(1)>\beta^{\prime}(1)$ and

$$
w u^{\prime}(1)>w \beta^{\prime}(1)
$$

For the case that $\lim _{t \rightarrow 1^{-}} \int_{1 / 2}^{t} h(s) d s=\infty$,

$$
\begin{aligned}
& w \beta_{1}^{\prime}(1)=\lim _{t \rightarrow 1^{-}} w(t) \beta_{1}^{\prime}(t) \\
& =\lim _{t \rightarrow 1^{-}} \frac{1}{\varphi_{p}^{-1}\left(\int_{1 / 2}^{t} h(s) d s\right)} \\
& \times\left[-\varphi_{p}^{-1}\left(-\alpha\left(\frac{a^{p-1}}{\|e\|_{\infty}^{p-1}} h\right)+\frac{a^{p-1}}{\|e\|_{\infty}^{p-1}} \int_{1 / 2}^{t} h(s) d s\right)\right] \\
& =\lim _{t \rightarrow 1^{-}}-\varphi_{p}^{-1}\left(-\frac{\alpha\left(\left(a^{p-1} /\|e\|_{\infty}^{p-1}\right) h\right)}{\int_{1 / 2}^{t} h(s) d s}+\frac{a^{p-1}}{\|e\|_{\infty}^{p-1}}\right) \\
& =\varphi_{p}^{-1}\left(-\frac{a^{p-1}}{\|e\|_{\infty}^{p-1}}\right) \text {. }
\end{aligned}
$$

From the monotonicity of $f$ and choice of $\lambda$ with help of L'Hospital's rule, we have

$$
\begin{aligned}
w u^{\prime}(1)= & \lim _{t \rightarrow 1^{-}} w(t) u^{\prime}(t) \\
= & \lim _{t \rightarrow 1^{-}} \frac{1}{\varphi_{p}^{-1}\left(\int_{1 / 2}^{t} h(s) d s\right)} \\
& \times\left[-\varphi_{p}^{-1}\left(-\alpha(\lambda h f(u))+\int_{1 / 2}^{t} \lambda h(s) f(u(s)) d s\right)\right] \\
= & \lim _{t \rightarrow 1^{-}}-\varphi_{p}^{-1}\left(-\frac{\alpha(\lambda h f(u))}{\int_{1 / 2}^{t} h(s) d s}+\frac{\int_{1 / 2}^{t} \lambda h(s) f(u(s)) d s}{1 / 2} h(s) d s\right. \\
= & \varphi_{p}^{-1}\left(-\lim _{t \rightarrow 1^{-}} \frac{\int_{1 / 2}^{t} \lambda h(s) f(u(s)) d s}{\int_{1 / 2}^{t} h(s) d s}\right) \\
= & \varphi_{p}^{-1}\left(-\lim _{t \rightarrow 1^{-}} \frac{\lambda h(t) f(u(t))}{h(t)}\right) \\
& =\varphi_{p}^{-1}\left(-\lim _{t \rightarrow 1^{-}} \lambda f(u(t))\right)=\varphi_{p}^{-1}(-\lambda f(0)) \\
& >\varphi_{p}^{-1}\left(-\frac{a^{p-1} f(0)}{\left.f(a)\|e\|_{\infty}^{p-1}\right)}\right. \\
& \left.\|e\|_{\infty}^{p-1}\right)=w \beta_{1}^{\prime}(1) .
\end{aligned}
$$

Thus we proved that $\beta_{1}$ is a strict upper solution of $\left(P_{\lambda}\right)$. Now, since $\lambda>b^{p-1} C / f(b)\|e\|_{\infty}^{p-1}$, we may choose $\lambda^{*}$ satisfying $b^{p-1} C / f(b)\|e\|_{\infty}^{p-1}<\lambda^{*}<\lambda$; then since $\lambda^{*} \underline{h} f(b) / 4^{p} b^{p-1}>1$, 
we may choose $k, j>1$ such that $1<(k j)^{p-1}<\lambda^{*} \underline{h} f(b) /$ $4^{p} b^{p-1}$. Let $\alpha_{2}$ be the solution of

$$
\begin{gathered}
\varphi_{p}\left(\alpha_{2}^{\prime}(t)\right)^{\prime}+\lambda^{*} \underline{h} f(v(t))=0, \quad t \in(0,1), \\
\alpha_{2}(0)=0=\alpha_{2}(1),
\end{gathered}
$$

where $v(t)=b \gamma(t)$ when

$$
\gamma(t)= \begin{cases}1-\left(1-(4 t)^{k}\right)^{j}, & \text { if } 0 \leq t \leq \frac{1}{4} \\ 1, & \text { if } \frac{1}{4} \leq t \leq \frac{1}{2}\end{cases}
$$

and $\gamma(t)=\gamma(1-t)$, for $t \in(1 / 2,1]$. We note that $\left|v^{\prime}(t)\right| \leq 4 k j b$ and let us show that $v(t) \leq \alpha_{2}(t)$ for $0 \leq t \leq 1 / 2$. It is clear that $\alpha_{2}^{\prime}(t) \geq 0=v^{\prime}(t)$ for $1 / 4 \leq t \leq 1 / 2$. We note that $\alpha_{2}^{\prime}(1 / 2)=0$ from the symmetry of $v$. For $0 \leq t \leq 1 / 4$, by integrating (63) from $t$ to $1 / 2$, from the choice of $\lambda^{*}$ and $C$, we have

$$
\begin{aligned}
\alpha_{2}^{\prime}(t) & =\varphi_{p}^{-1}\left(\int_{t}^{1 / 2} \lambda^{*} \underline{h} f(v(s)) d s\right) \\
& \geq \varphi_{p}^{-1}\left(\int_{1 / 4}^{1 / 2} \lambda^{*} \underline{h} f(w(s)) d s\right) \\
& =\left(\lambda^{*} \underline{h} f(b) \frac{1}{4}\right)^{1 /(p-1)}>4 k j b \geq v^{\prime}(t) .
\end{aligned}
$$

Thus $\alpha_{2}(t)>v(t)$ for $0<t \leq 1 / 2$ and it is clear that $\alpha_{2}(t)>$ $v(t)$ for $0<t \leq 1$, by the symmetry of $\alpha_{2}$ and $v$. From the monotonicity of $f$, we have

$$
\begin{aligned}
-\varphi_{p}\left(\alpha_{2}^{\prime}(t)\right)^{\prime} & =\lambda^{*} \underline{h} f(v(t))<\lambda h(t) f(v(t)) \\
& \leq \lambda h(t) f\left(\alpha_{2}(t)\right) .
\end{aligned}
$$

This implies that $\alpha_{2}$ is a lower solution of $\left(P_{\lambda}\right)$ and by using the similar argument as of $\beta_{1}$, we can show that $\alpha_{2}(t)<u(t)$ for all solution $u$ of $\left(P_{\lambda}\right)$ such that $u \geq \alpha_{2}$. Now to show that $\alpha_{2}$ is a strict lower solution of $\left(P_{\lambda}\right)$, we need to show that $w u^{\prime}(0)>w \alpha_{2}^{\prime}(0)$ and $w u^{\prime}(1)<w \alpha_{2}^{\prime}(1)$. For the case that $\lim _{t \rightarrow 0^{+}} \int_{t}^{1 / 2} h(s) d s<\infty, w u^{\prime}(1)<w \alpha_{2}^{\prime}(1)$ can be proved by similar argument as of $\beta_{1}$. Now, for the case of $\lim _{t \rightarrow 0^{+}} \int_{t}^{1 / 2} h(s) d s=\infty$, from (65) and $\lim _{t \rightarrow 0^{+}} w(t)=0$, we have $w \alpha_{2}^{\prime}(0)=\lim _{t \rightarrow 0^{+}} w(t) \alpha_{2}^{\prime}(t)=0$ and

$$
\begin{aligned}
w u^{\prime}(0)= & \lim _{t \rightarrow 0^{+}} w(t) u^{\prime}(t) \\
= & \lim _{t \rightarrow 0^{+}} \frac{1}{\varphi_{p}^{-1}\left(\int_{t}^{1 / 2} h(s) d s\right)} \\
& \times\left[\varphi_{p}^{-1}\left(\alpha(\lambda h f(u))+\int_{t}^{1 / 2} \lambda h(s) f(u(s)) d s\right)\right] \\
= & \varphi_{p}^{-1}\left(\lim _{t \rightarrow 0^{+}} \frac{\lambda h(t) f(u(t))}{h(t)}\right)=\lambda f(0)>0 \\
= & w \alpha_{2}^{\prime}(0) .
\end{aligned}
$$

Similarly, we can prove $w u^{\prime}(1)<w \alpha_{2}^{\prime}(1)$ and thus $u>\alpha_{2}$ for all solution $u$ of $\left(P_{\lambda}\right)$ such that $u \geq \alpha_{2}$. This implies that $\alpha_{2}$ is a strict lower solution of $\left(P_{\lambda}\right)$. Since $\left\|\alpha_{2}\right\|_{\infty} \geq\|v\|_{\infty}=b>$ $a=\left\|\beta_{1}\right\|_{\infty}$, there exists $t_{0} \in(0,1)$ such that $\alpha_{2}\left(t_{0}\right)>\beta_{1}\left(t_{0}\right)$. Define

$$
\beta_{2}=\lambda M \frac{e}{\|e\|_{\infty}} .
$$

Then from $\left(F_{1}\right)$, there exists sufficiently large $M \gg 1$ such that

$$
\frac{f(\lambda M)}{(\lambda M)^{p-1}}<\frac{\lambda}{\|e\|_{\infty}^{p-1}}
$$

and $\beta_{2}>\alpha_{2}, \beta_{2}>\beta_{1}$. Thus we have

$$
\begin{aligned}
-\varphi_{p}\left(\beta_{2}^{\prime}(t)\right)^{\prime} & =-\frac{(\lambda M)^{p-1} \varphi_{p}\left(e^{\prime}(t)\right)^{\prime}}{\|e\|_{\infty}^{p-1}} \\
& >\lambda h(t) f(\lambda M) \geq \lambda h(t) f\left(\beta_{2}(t)\right) .
\end{aligned}
$$

This implies that $\beta_{2}$ is an upper solution of $\left(P_{\lambda}\right)$ and the proof is complete by three solutions theorem.

\section{Example}

As an example, let us consider the following combustion model defined on an exterior domain:

$$
\begin{gathered}
-\Delta_{p} u=\lambda k(|x|) \exp \left(\frac{\alpha u}{\alpha+u}\right), \quad x \in \Omega, \\
\left.u\right|_{|x|=r_{0}}=0, \quad u \longrightarrow 0 \text { as }|x| \longrightarrow \infty,
\end{gathered}
$$

where $\Delta_{p} u=\operatorname{div}\left(|\nabla u|^{p-2} \nabla u\right), \Omega=\left\{x \in \mathbb{R}^{N}|r<| x \mid<\infty\right\}$, $1<p<N$, and $\alpha, \lambda>0$. Moreover $k \in L_{\text {loc }}^{1}\left(\left[r_{0}, \infty\right),(0, \infty)\right)$. For the radial solutions of $\left(E_{\lambda}\right)$, by changes of variables, $r=|x|, u(r)=u(|x|), t=\left(r / r_{0}\right)^{(-N+p) /(p-1)},\left(E_{\lambda}\right)$ can be transformed into $\left(P_{\lambda}\right)$ with

$$
h(t)=\left(\frac{p-1}{N-p}\right)^{p} r_{0}^{p} t^{-p(N-1) /(N-p)} k\left(r_{0} t^{-(p-1) /(N-p)}\right) .
$$

Let us define

$$
\begin{aligned}
\mathscr{K}=\left\{k \in L_{\mathrm{loc}}^{1}\left(\left[r_{0}, \infty\right)\right) \mid \int_{r_{0}}^{\infty} \varphi_{p}^{-1}\left(\tau^{1-N} \int_{r_{0}}^{\tau} r^{N-1} k(r) d r\right) d \tau\right. \\
<\infty\}, \\
\mathscr{K}_{1}=\left\{k \in L_{\mathrm{loc}}^{1}\left(\left[r_{0}, \infty\right)\right) \mid \int_{r_{0}}^{\infty} r^{N-1} k(r) d r<\infty\right\} ;
\end{aligned}
$$

then it is easy to check $\mathscr{K}_{1} \varsubsetneqq \mathscr{K}$ and if $k \in \mathscr{K} \backslash \mathscr{K}_{1}$, then corresponding $h$ in (71) satisfies $h \in \mathscr{H} \backslash L^{1}(0,1)$. 
As an example of $k$, take $k(r)=r^{\delta}$ for $-N<\delta<-p$; then $k \in \mathscr{K} \backslash \mathscr{K}_{1}$. Moreover, $h$ in (71) can be calculated as $h(t)=C_{2} t^{\rho}$, for some $C_{2}>0$ and $\rho$ given as

$$
\rho=\frac{-p(N+\delta)+(p+\delta)}{N-p}
$$

and we see that $h \in \mathscr{H} \backslash L^{1}(0,1)$, when $-N<\delta<-p$.

Finally, we have a multiplicity result of positive solutions for combustion model $\left(E_{\lambda}\right)$.

Corollary 12. Assume $k \in \mathscr{K} \backslash \mathscr{K}_{1}$. If $\alpha$ is sufficiently large, then $\left(E_{\lambda}\right)$ has at least three positive radial solutions for $\lambda \in$ $\left(I_{1}, I_{2}\right)$, where

$$
I_{1}=\frac{4^{p} \alpha^{p-1}}{\underline{h} \exp (\alpha / 2)}, \quad I_{2}=\frac{1}{\exp (\alpha /(\alpha+1))\|e\|_{\infty}^{p-1}} .
$$

Proof. Let $f(u)=\exp (\alpha u /(\alpha+u))$ and $a=1, b=\alpha$. Then since $f$ is nondecreasing and

$$
\frac{1 / f(1)}{\alpha^{p-1} / f(\alpha)}=\frac{1}{\alpha^{p-1}} \frac{f(\alpha)}{f(1)}=\frac{1}{\alpha^{p-1}} \exp \left(\frac{\alpha}{2}-\frac{\alpha}{\alpha+1}\right) \rightarrow \infty
$$

as $\alpha \rightarrow \infty$, all hypotheses of Theorem 11 are satisfied for sufficiently large $\alpha$ and we get the conclusion.

\section{Conflict of Interests}

The authors declare that there is no conflict of interests regarding the publication of this paper.

\section{Acknowledgments}

This research was supported by Basic Science Research Program through the National Research Foundation of Korea (NRF) funded by the Ministry of Education, Science and Technology (no. 2012005767). This research was supported by Basic Science Research Program through the National Research Foundation of Korea (NRF) funded by the Ministry of Education, Science and Technology (2012R1A1A1011225).

\section{References}

[1] A. K. Ben-Naoum and C. de Coster, "On the existence and multiplicity of positive solutions of the $p$-Laplacian separated boundary value problem," Differential and Integral Equations, vol. 10, pp. 1093-1112, 1997.

[2] G. Bonannao, "Existence of three solutions for a two point boundary value problem," Applied Mathematics Letters, vol. 13, no. 5, pp. 53-57, 2000.

[3] J. R. Graef, S. Heidarkhani, and L. Kong, "A critical points approach for the existence of multiple solutions of a Dirichlet quasilinear system," Journal of Mathematical Analysis and Applications, vol. 388, no. 2, pp. 1268-1278, 2012.

[4] B. Ricceri, "A three critical points theorem revisited," Nonlinear Analysis: Theory, Methods and Applications, vol. 70, no. 9, pp. 3084-3089, 2009.
[5] B. Ricceri, "On a three critical points theorem," Archiv der Mathematik, vol. 75, no. 3, pp. 220-226, 2000.

[6] G. L. Karakostas, "Triple positive solutions for the $\Phi$-Laplacian when $\Phi$ is a sup-multiplicative-like function," Electronic Journal of Differential Equations, vol. 69, pp. 1-13, 2004.

[7] J. Li and J. Wang, "Triple positive solutions for a type of secondorder singular boundary problems," Boundary Value Problems, vol. 2010, Article ID 376471, 2010.

[8] J. Ren, W. Ge, and B. Ren, "Existence of three positive solutions for quasi-linear boundary value problem," Acta Mathematicae Applicatae Sinica. English Series, vol. 21, no. 3, pp. 353-358, 2005.

[9] E. K. Lee and Y. H. Lee, "A result on three solutions theorem and its application to $p$-Laplacian systems with singular weights," Boundary Value Problems, vol. 2012, article 63, 20 pages, 2012.

[10] I. Sim and Y. H. Lee, "A new solution operaor of one dimensional $p$-Laplacian with a sign-changing weight and its application," Abstract and Applied Analysis, vol. 2012, Article ID 243740, 15 pages, 2012.

[11] E. Ko, E. K. Lee, and R. Shivaji, "Multiplicity results for classes of singular problems on an exterior domain," Discrete and Continuous Dynamical Systems, vol. 33, no. 11/12, pp. 5153-5166, 2013. 


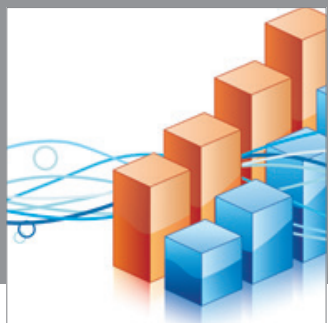

Advances in

Operations Research

mansans

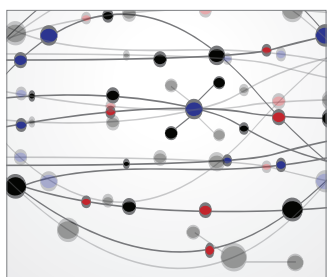

The Scientific World Journal
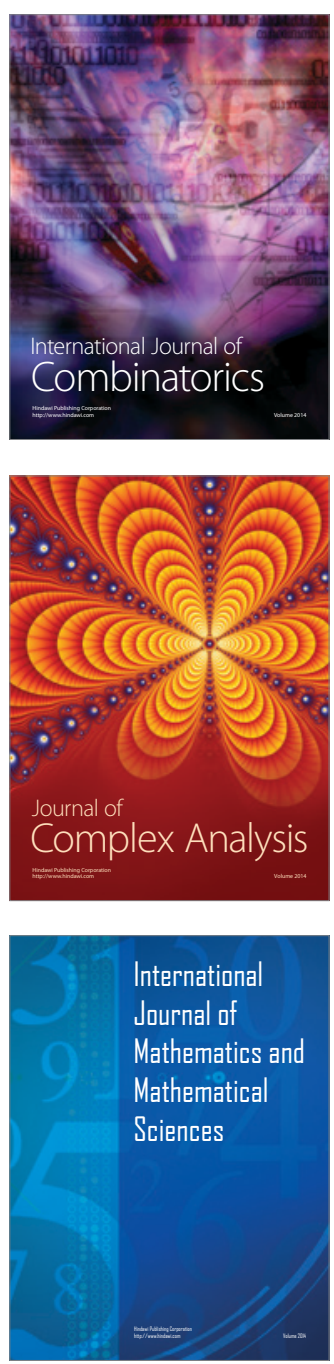
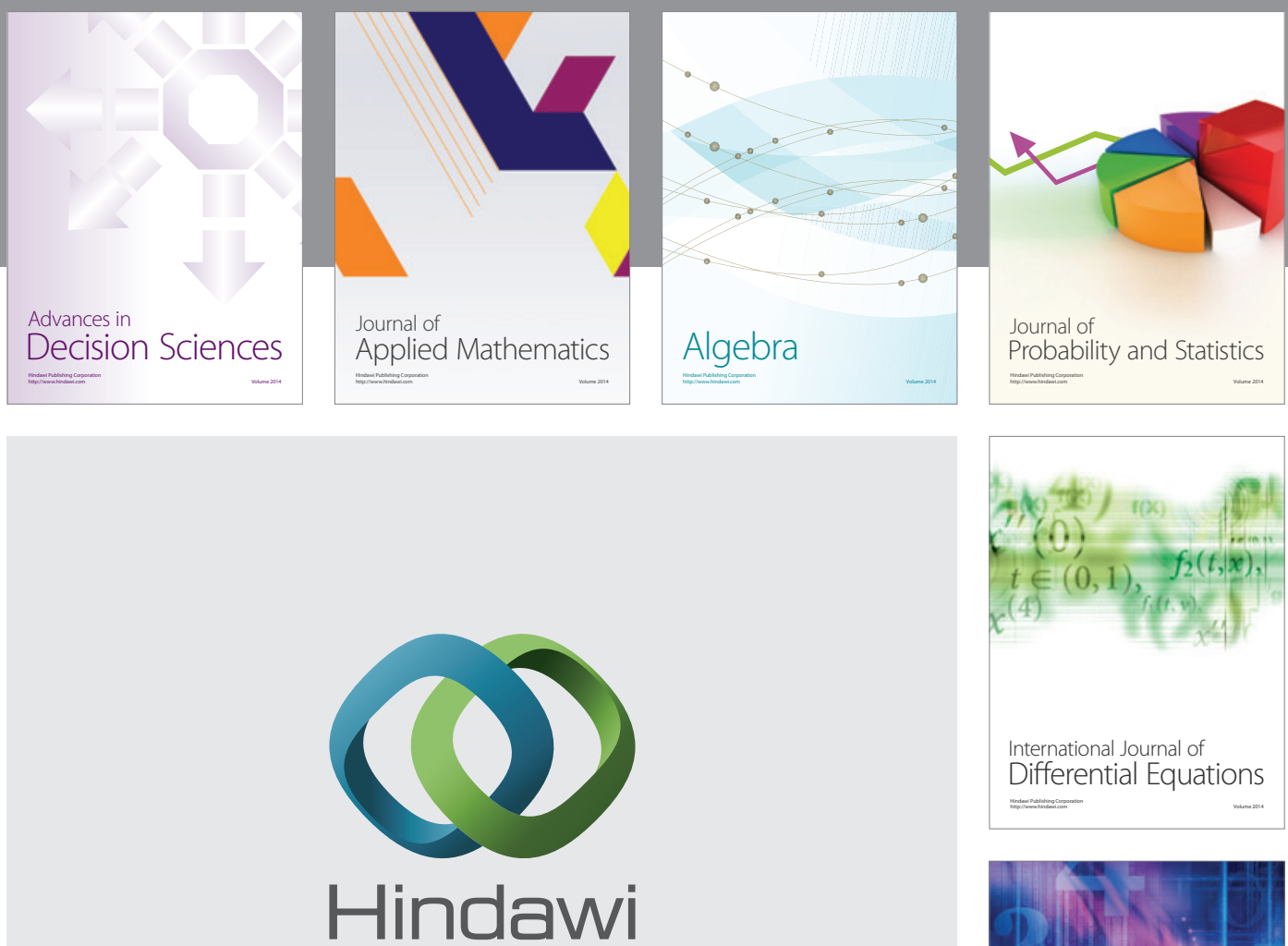

Submit your manuscripts at http://www.hindawi.com
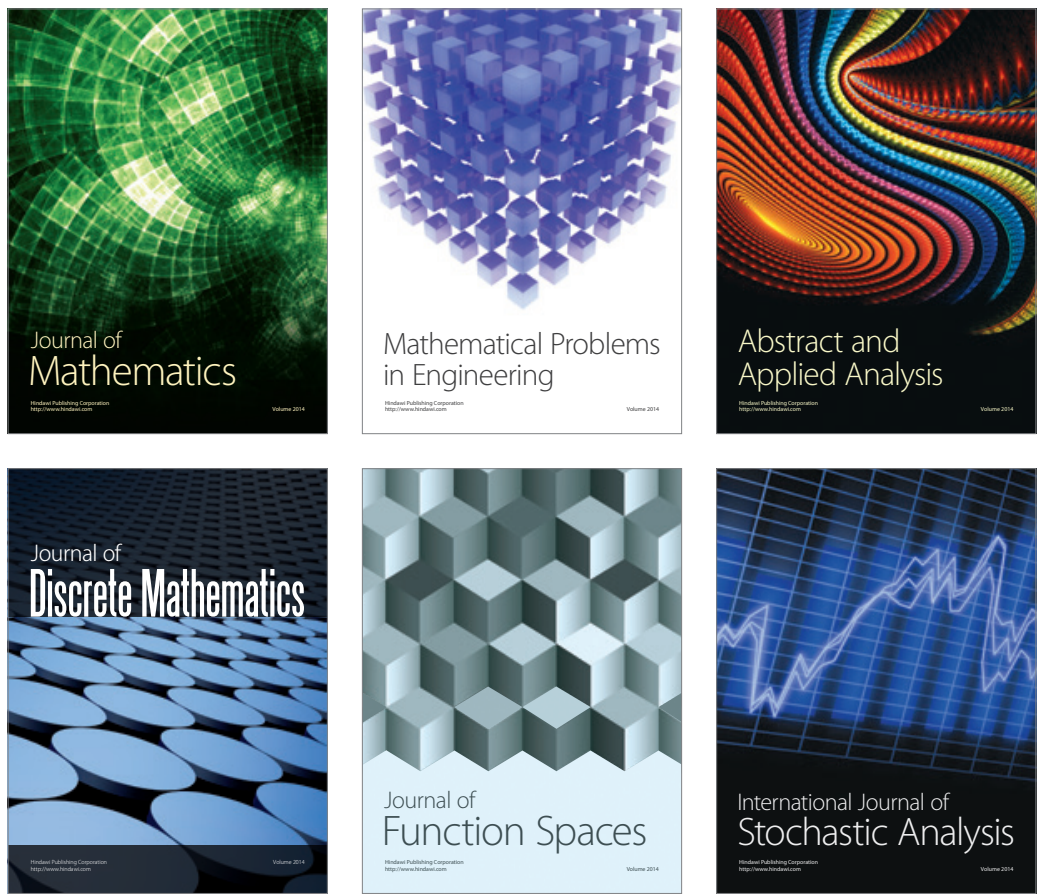

Journal of

Function Spaces

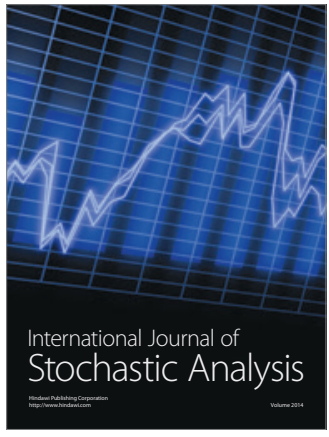

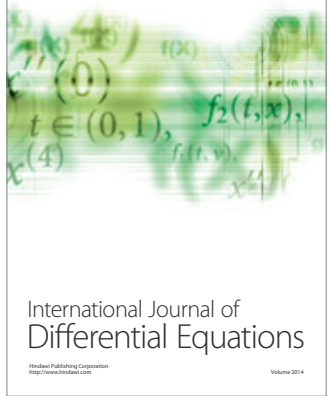
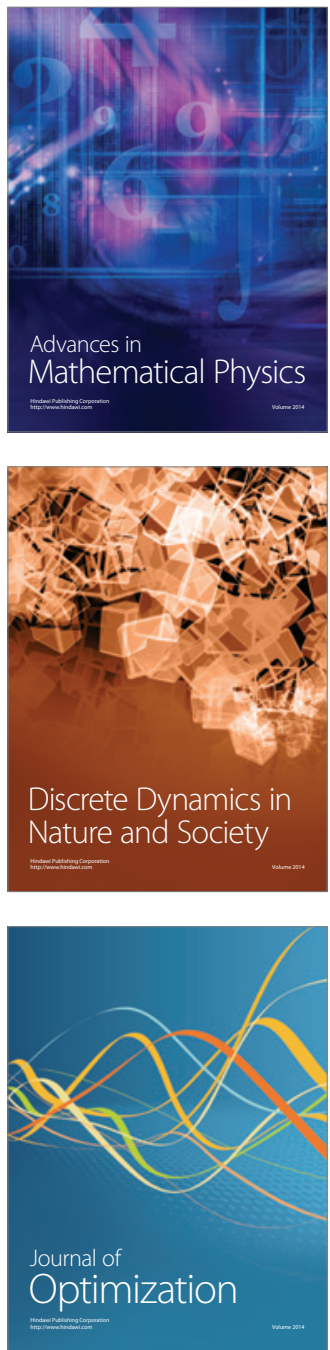